\title{
AN INNOVATIVE STUDY ON SUM OF POWERS OF INTEGERS
}

\section{B.Mahaboob $^{\text {a }}$, Y.Hari Krishna ${ }^{\text {b }}$, B.Sivaram ${ }^{c}$, C.Narayana $^{d}$, P.SreehariReddy ${ }^{\mathrm{e}}$ and G.Balaji Prakash $^{\mathrm{f}}$}

${ }^{a,}{ }^{\mathrm{f}}$ Department of Mathematics, KoneruLakshmaiah Education Foundation, Vaddeswaram, Guntur A.P. India.

${ }^{b}$ Department of Mathematics, ANURAG Engineering College, Ananthagiri (v), Kodad,Suryapet,Telangana-508 206

${ }^{c}$ Department of Mathematics, KoneruLakshmaiah Education Foundation, Aziznagar, HyderabadA.P. India

${ }^{d}$ Department of Mathematics, SriHarsha Institute of PG Studies, SPSR Nellore(Dt),A.P., India.

${ }^{e}$ Department of Mathematics, NBKR S\&A College,Vidyanagar,SPSR Nellore(Dt),A.P., India

Article History: Received: 11 January 2021; Accepted: 27 February 2021; Published online: 5 April 2021

\begin{abstract}
The generalization of sum of integral powers of first n-natural numbers has been an interesting problem among the researchers in Analytical Number Theory for decades. This research article mainly focuses on the derivation of generalized result of this sum. More explicit formula has been derived in order to get the sum of any arbitrary integral powers of first $n$ natural numbers. Furthermore by using the fundamental principles of Combinatorics and Linear Algebra an attempt has been made to answer an interesting question namely: Is the sum of integral powers of natural numbers a unique polynomial? As a result it is depicted that this sum always equals a unique polynomial over natural numbers. Moreover some properties of the coefficients of this polynomial are derived.More importantly a recurrence relation which can give the formulas for sum of any positive integral powers of first $n$-natural numbers has been proposed and it is strongly believed that this recurrence relation is the most significant thing in this entire discussion
\end{abstract}

Key words: Lower triangular matrix, Full rank, Simultaneous non-homogeneous linear equations, Echelon matrix, Consistency, Rank test

\section{Introduction}

Formulas of sum of integers were first given in generalizable from in west by Thomas Harrot (1560-1621) of England. At about the same time Johann Faulhaber (1580-1635) of Germany gave formulas for these sums upto the $17^{\text {th }}$ power for higher than anyone before him, but hid did not make clear to generalize them. In this article an attempt has been made to give the generalized result. First the mathematical modeling to the evaluation of where $\mathrm{p}=1,2,3, \ldots$ is made as follows.

$$
\begin{aligned}
& 1^{2}+2^{2}+3^{2}+\cdots+n^{2}=\sum_{r=1}^{n} r^{2} \\
& 2^{2}+3^{2}+\cdots+n^{2}+(n+1)^{2}=\sum_{r=1}^{n}(r+1)^{2}
\end{aligned}
$$

On subtraction $\quad(n+1)^{2}-1^{2}=\sum_{r=1}^{r=1}(r+1)^{2}-$

$$
\begin{aligned}
& n^{2}+2 n=\sum_{r=1}^{n}(2 r+1) \\
& n^{2}+2 n=2 \sum^{n} n+n \\
& \sum_{n=1}^{n} n=\frac{n^{2}+n}{2}=\frac{1}{2} n^{2}+\frac{1}{2} n \\
& 1^{3}+2^{3}+3^{3}+\cdots+n^{3}=\sum_{r=1}^{n} r^{3}
\end{aligned}
$$$$
2^{3}+3^{3}+\cdots+n^{3}+(n+1)^{3}=\sum_{r=1}^{n}(r+1)^{3}
$$$$
\text { On subtraction } \quad n^{3}+3 n^{2}+3 n=\sum_{r=1}^{n=1}\left(3 r^{2}+3 r+\right.
$$$$
3 \sum n^{2}+3 \sum n=n^{3}+3 n^{2}+2 n
$$$$
3 \sum n^{2}+\frac{3}{2} n^{2}+\frac{3}{2} n=n^{3}+3 n^{2}+2 n
$$ 
$3 \sum n^{2}=n^{3}+\frac{3 n^{2}}{2}+\frac{n}{2}$
$\sum n^{2}=\frac{n^{3}}{3}+\frac{n^{2}}{2}+\frac{n}{6}$

$1^{4}+2^{4}+3^{4}+\cdots+n^{4}=\sum_{r=1}^{n} r^{4}$

$2^{4}+3^{4}+\cdots+n^{4}+(n+1)^{4}=\sum_{r=1}^{n}(r+1)^{4}$

On subtraction $\quad(n+1)^{4}-1^{4}=\sum_{r=1}^{n=1}\left(4 r^{3}+6 r^{2}+4 r+\right.$

$n^{4}+4 n^{3}+6 n^{2}+4 n=4 \sum n^{3}+6 \sum n^{2}+4 \sum n+\sum 1$

$n^{4}+4 n^{3}+6 n^{2}+4 n=4 \sum n^{3}+6\left(\frac{n^{3}}{3}+\frac{n^{2}}{2}+\frac{n}{6}\right)+4\left(\frac{n^{2}}{2}+\frac{n}{2}\right)+n$

$n^{4}+4 n^{3}+6 n^{2}+4 n=4 \sum n^{3}+2 n^{3}+3 n^{2}+n+2 n^{2}+2 n+n$

$n^{4}+2 n^{3}+n^{2}=4 \sum n^{3}$

$\sum n^{3}=\frac{n^{4}}{4}+\frac{n^{3}}{2}+\frac{n^{2}}{2}$

From the above observations one can assume that

$\Sigma$ is a polynomial of degree $p+1$ in $n$ over natural numbers and this polynomial is free of constant terms.

2. Existence and Uniqueness of Polynomial

Let $\sum_{m=1}^{n} m^{p}=1^{p}+2^{p}+3^{p}+\ldots \ldots \ldots \ldots \ldots+n^{p}=\sum$

$p \in W=\{0,1,2,3 \ldots \ldots\}$

One can make the assumption as follows

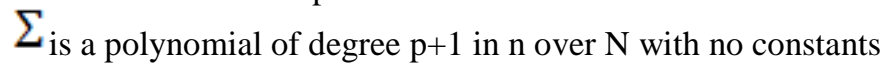

That is

$$
\sum_{m=1}^{n} n^{p}=\sum n^{p}=k_{0} n^{p+1}+k_{1} n^{p}+k_{2} n^{p-1}+k_{3} n^{k-2}+\cdots+k
$$

where $k_{0}, k_{1}, k_{2}, \ldots \ldots \ldots$ are unknown coefficients.

To prove this one can use direct proof in the sense that if $\quad k_{0}, k_{1}, k_{2}, \ldots \ldots \ldots$ exist then the above statement is valid

Now one can have

$$
1^{p}+2^{p}+3^{p}+\cdots \ldots \ldots \ldots \ldots+n^{p}=k_{0} n^{p+1}+k_{1} n^{p}+k_{2} n^{p-1}+\cdots+k_{(2)}
$$

Replacing $\mathrm{n}$ by $(\mathrm{n}+1)$

$$
\begin{aligned}
1^{p}+2^{p}+3^{p} & +\cdots \ldots \ldots \ldots+n^{p}+(n+1)^{p} \\
& =k_{0}(n+1)^{p+1}+k_{1}(n+1)^{p}+k_{2}(n+1)^{p-1}+\cdots+k_{p}(n+1)
\end{aligned}
$$

Subtracting (2) from (3)

$$
\begin{gathered}
(n+1)^{p}=k_{0}\left[(n+1)^{p+1}-n^{p+1}\right]+k_{1}\left[(n+1)^{p}-n^{p}\right]+k_{2}\left[(n+1)^{p-1}-n^{p-1}\right] \\
+\cdots+k_{p-1}\left[(n+1)^{2}-n^{2}\right]+k_{p}[(n+1)-n] \\
p_{c_{0}} n^{p}+p_{c_{1}} n^{p-1}+p_{c_{2}} n^{p-2}+\cdots+p_{c_{p-1}} n+p_{c_{p}}= \\
k_{0}\left[(p+1)_{c_{1}} n^{p}+(p+1)_{c_{2}} n^{p-1}+\cdots+(p+1)_{c_{p}} n+(p+1)_{c_{[p+1)}}\right] \\
\quad k_{1}\left[p_{c_{1}} n^{p-1}+p_{c_{2}} n^{p-2}+\cdots+p_{c_{p-1}} n+p\right. \\
+k_{2}\left[(p-1)_{c_{1}} n^{p-2}+(p-1)_{c_{2}} n^{p-3}+\cdots+(p-1)_{c_{p-2}} n+(p-1)_{c_{p}}\right.
\end{gathered}
$$


$+\quad k_{p-1}\left[2_{c_{1}} n+2_{c_{2}}\right]+k_{p}[1$

Comparing the coefficients of $n^{p}, n^{p-1}, n^{p-2}, \ldots \ldots \ldots$, constants

$p_{c_{0}}=k_{0}(p+1)_{c_{1}}$

$p_{c_{1}}=k_{0}(p+1)_{c_{2}}+k_{1} p_{c_{1}}$

$p_{c_{2}}=k_{0}(p+1)_{c_{3}}+k_{1} p_{c_{2}}+k_{2}(p-1)_{c_{1}}$

$p_{c_{3}}=k_{0}(p+1)_{c_{4}}+k_{1} p_{c_{3}}+k_{2}(p-1)_{c_{2}}+k_{3}(p-2)_{c_{1}}$

$p_{c_{p-1}}=k_{0}(p+1)_{c_{p}}+k_{1} p_{c_{p-1}}+k_{2}(p-1)_{c_{(p-2)}}+\cdots+k_{p-1} 2_{c_{1}}$

$p_{c_{p}}=k_{0}(p+1)_{c_{(p+1)}}+k_{1} p_{c_{p}}+k_{2}(p-1)_{c_{(p-1)}}+\cdots+k_{p} 1_{c_{1}}$

These are $(\mathrm{p}+1)$ simultaneous non homogeneous linear equations in $(\mathrm{p}+1)$ unknowns $\quad k_{0}, k_{1}, \ldots$.

From the last one it is obvious that $k_{0}+k_{1}+k_{2}+\cdots+k_{p}=$

From the first one, one can get $\quad k_{0}=\frac{-}{p}$

Writing the above system in matrix notation

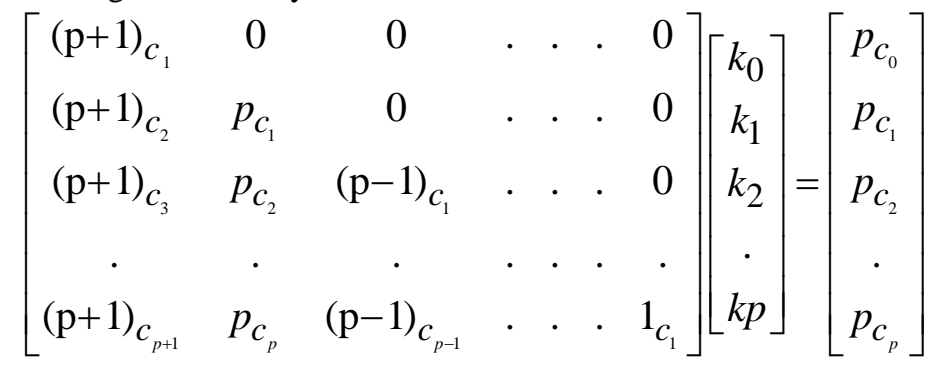

$M X=N$

$X=\left[\begin{array}{c}k_{0} \\ k_{1} \\ k_{2} \\ \cdot \\ k p\end{array}\right]$, a column vector

$N=\left[\begin{array}{c}p_{c_{0}} \\ p_{c_{1}} \\ p_{c_{2}} \\ \cdot \\ p_{c_{p}}\end{array}\right]$, a column vector

$\mathrm{M}$ is of full rank and $\quad \rho(M)=p \dashv$ 
$\mathrm{M}$ is lower triangular matrix.

$|M|=(p+1) ! \neq 0$

Now the consistency of this system is to be examined through rank test

Number of unknowns $=\mathrm{p}+1=$ Number of equations

$$
M \square\left[\begin{array}{cccccccc}
(\mathrm{p}+1)_{c_{p+1}} & p_{c_{p}} & (\mathrm{p}-1)_{c_{p-1}} & . & . & . & 2_{c_{2}} & 1_{c_{1}} \\
(\mathrm{p}+1)_{c_{p}} & p_{c_{p-1}} & (\mathrm{p}-1)_{c_{p-2}} & . & . & . & 2_{c_{1}} & 0 \\
. & . & . & . & . & . & . & . \\
(\mathrm{p}+1)_{c_{3}} & p_{c_{2}} & (\mathrm{p}-1)_{c_{1}} & . & . & . & 0 & 0 \\
(\mathrm{p}+1)_{c_{2}} & p_{c_{1}} & 0 & . & . & . & 0 & 0 \\
(\mathrm{p}+1)_{c_{1}} & 0 & 0 & . & . & . & 0 & 0
\end{array}\right]
$$

$\mathrm{M}$ is an Echelon matrix

Number of non-zero rows in $\quad M=p+1=\rho($

$$
\begin{aligned}
& M \square\left[\begin{array}{ccccccccc}
(\mathrm{p}+1)_{c_{p+1}} & p_{c_{p}} & (\mathrm{p}-1)_{c_{p-1}} & . & . & . & 2_{c_{2}} & 1_{c_{1}} & p_{c_{p}} \\
(\mathrm{p}+1)_{c_{p}} & p_{c_{p-1}} & (\mathrm{p}-1)_{c_{p-2}} & . & . & . & 2_{c_{1}} & 0 & p_{c_{p-1}} \\
\cdot & \cdot & . & . & . & . & . & . & \cdot \\
(\mathrm{p}+1)_{c_{3}} & p_{c_{2}} & (\mathrm{p}-1)_{c_{1}} & . & . & . & 0 & 0 & p_{c_{2}} \\
(\mathrm{p}+1)_{c_{2}} & p_{c_{1}} & 0 & . & . & . & 0 & 0 & p_{c_{1}} \\
(\mathrm{p}+1)_{c_{1}} & 0 & 0 & . & . & . & 0 & 0 & p_{c_{0}}
\end{array}\right] \\
& \rho([M N])=p+1
\end{aligned}
$$

Since $\rho(M)=\rho([M N])=p t_{\text {the system is consistent. }}$

Further common rank = number of unknowns

Hence the above system possesses unique solution.

Consequently $k_{0}, k_{1}, \ldots$ exist uniquely.

\section{Computing the coefficients}

From the above matrix system one can get

$$
\begin{aligned}
& (p+1)_{c_{1}} k_{0}=p_{c_{0}} \\
& k_{0}=\frac{1}{p+1} \\
& (p+1)_{c_{3}} k_{0}+p_{c_{1}} k_{1}=p_{c_{1}} \\
& \frac{(p+1) p}{1.2} \frac{1}{p+1}+p k_{1}=p \\
& k_{1}=\frac{1}{2} \\
& (p+1)_{c_{3}} k_{0}+p_{c_{2}} k_{1}+(p-1)_{c_{1}} k_{2}=p_{c_{2}} \\
& \frac{(p+1) p(p-1)}{1} \frac{1}{p+1}+\frac{p(p-1)}{1.2} \frac{1}{2}+(p-1) k_{2}=\frac{p(p-1)}{2} \\
& \frac{p}{6}+\frac{p}{4}+k_{2}=\frac{p}{2} \\
& k_{2}=\frac{p}{12} \\
& (p+1)_{c_{4}} k_{0}+p_{c_{3}} k_{1}+(p-1)_{c_{2}} k_{2}+(p-2)_{c_{1}} k_{3}=p_{c_{3}}
\end{aligned}
$$




$$
\begin{aligned}
& \frac{(p+1) p(p-1)(p-2)}{1.2 .3 .4} \frac{1}{p+1}+\frac{p(p-1)(p-2)}{1.2 .3} \frac{1}{2}+\frac{(p-1)(p-2)}{1.2} \frac{p}{12}+(p-2) k_{3} \\
& =\frac{p(p-1)(p-2)}{1.2 .3} \\
& \frac{p(p-1)}{24}+\frac{p(p-1)}{12}+\frac{p(p-1)}{24}+k_{3}=\frac{p(p-1)}{6} \\
& k_{3}=0 \\
& (p+1)_{5} k_{0}+p_{c_{4}} k_{1}+(p-1)_{c_{3}} k_{2}+(p-2)_{c_{2}} k_{3}+(p-3)_{c_{1}} k_{4}=p_{c_{4}} \\
& \frac{(p+1) p(p-1)(p-2)(p-3)}{p+1}+\frac{p(p-1)(p-2)(p-3)}{1}+\frac{1}{2}+1.2 .3 .4 \\
& \frac{(p-1)(p-2)(p-3)}{1.2} \frac{p}{12}+\frac{(p-2)(p-3)}{1.2 .3} 0+(p-3) k_{4}=\frac{p(p-1)(p-2)(p-3)}{1.2} \\
& \frac{120}{p(p-1)(p-2)}+\frac{p(p-1)(p-2)}{48}+\frac{p(p-1)(p-2)}{72}+k_{4}=\frac{p(p-1)(p-2)}{24} \\
& k_{4}=p(p-1)(p-2)\left(\frac{1}{24}-\frac{1}{72}-\frac{1}{120}-\frac{1}{48}\right) \\
& k_{4}=\frac{-p(p-1)(p-2)}{720}
\end{aligned}
$$

In this manner on can get $\quad k_{5}, k_{6}, k_{7} \ldots$

It is interesting to observe that is a polynomial of degree $r-1$ in $p$ where $r=0,1,2,3, \ldots$

4. Applications of coefficients

$$
\begin{aligned}
& \sum_{m=1}^{n} m^{p}=k_{0} n^{p+1}+k_{1} n^{p}+k_{2} n^{p-1}+k_{3} n^{p-2}+\cdots+k_{p} n \\
& \text { For } p=1, \quad k_{0}=\frac{1}{p+1}=\frac{1}{2}
\end{aligned}
$$

$k_{1}=\frac{1}{2}$

So, $\quad \sum_{m=1}^{n} m^{p}=\sum n=1+2+3+\cdots \ldots \ldots+n=\frac{1}{2} n^{2}+$

For $\mathrm{p}=2 \quad k_{0}=\frac{1}{p+1}=$

$k_{1}=\frac{1}{2}$

$k_{2}=\frac{p}{12}=\frac{2}{12}=\frac{1}{6}$

So, $\quad \sum_{m=1}^{n} m^{p}=\sum n^{2}=1^{2}+2^{2}+3^{2}+\cdots \ldots \ldots+n^{2}=\frac{1}{3} n^{3}+\frac{1}{2} n^{2}+$

For $\mathrm{p}=3$.

$$
k_{0}=\frac{1}{p+1}=
$$

$k_{1}=\frac{1}{2}$

$k_{2}=\frac{p}{12}=\frac{3}{12}=\frac{1}{4}$

$k_{3}=0$

So,

$$
\sum_{m=1}^{n} m^{p}=\sum n^{3}=1^{3}+2^{3}+3^{3}+\cdots \ldots \ldots+n^{3}=\frac{1}{4} n^{4}+\frac{1}{2} n^{3}+\frac{1}{4}
$$

For $\mathrm{p}=4$

$k_{0}=\frac{1}{p+1}=\frac{1}{5}$ 
$k_{1}=\frac{1}{2}$

$k_{2}=\frac{p}{12}=\frac{4}{12}=\frac{1}{3}$

$k_{3}=0$

$k_{4}=\frac{-p(p-1)(p-2)}{720}=\frac{-4.3 .2}{720}=-\frac{1}{30}$

So, $\quad \sum_{m=1}^{n} m^{p}=\sum n^{4}=1^{4}+2^{4}+3^{4}+\cdots \ldots \ldots+n^{4}=\frac{1}{5} n^{5}+\frac{1}{2} n^{4}+\frac{1}{3} n^{3}-\frac{1}{3}$

Proceeding in this manner one can get $\sum n^{5}, \sum n^{6}, \sum n^{7}, \ldots$

All constants can be computed from the recurrence relation given by

$(p+1)_{c_{(i+1)}} k_{0}+p_{c_{i}} k_{1}+(p-1)_{c_{(i-1)}} k_{2}+\cdots+(p-i+1)_{c_{i}} k_{i}=p_{c_{i}}$

$i=0,1,2$

By substituting $\mathrm{i}=0,1,2,3 \ldots \ldots$ one can find $\quad k_{0}, k_{1}, k_{2}$

\section{Crammer's rule in the evaluation of constants}

The constants of the unique polynomial namely $k_{0}, k_{1}, k_{2} \ldots$ can be found by Crammer's rule as shown below.

$$
\begin{aligned}
& \Delta=|M|=(p+1) ! \neq 0 \\
& \Delta_{1}=\left|\begin{array}{cccccccc}
p_{c_{0}} & 0 & 0 & . & . & . & 0 & 0 \\
p_{c_{1}} & p_{c_{1}} & 0 & . & . & . & 0 & 0 \\
p_{c_{2}} & p_{c_{2}} & (p-1)_{c_{1}} & . & . & . & 0 & 0 \\
\cdot & \cdot & . & . & . & . & . & . \\
p_{c_{p-1}} & p_{c_{p-1}} & (p-1)_{c_{p-2}} & . & . & . & 2_{c_{1}} & 0 \\
p_{c_{p}} & p_{c_{p}} & (p-1)_{c_{p-1}} & . & . & . & 2_{c_{2}} & 1_{c_{1}}
\end{array}\right|
\end{aligned}
$$

$=$ The determinant obtained by replacing the first column of $\quad I \cdot$ by column vector $\mathrm{N}$

$=$ The determinant obtained by replacing the second column of $\quad I_{\text {by column vector } \mathrm{N}}$

$\Delta_{i}=$ The determinant obtained by replacing the $(\mathrm{p}+1)^{\mathrm{th}}$ column of $\quad \mathrm{I} \cdot$ by column vector $\mathrm{N}$

$k_{0}=\frac{\Delta_{1}}{\Delta} ; k_{1}=\frac{\Delta_{2}}{\Delta} ; \ldots \ldots \ldots k_{p}=\frac{\Delta_{p+1}}{\Delta}$

\section{Conclusions and Future Research}

In the above discussion a generalized result for sum of any arbitrary positive integral powers of first $n$-natural numbers has been derived by using the fundamentalconcepts in Combinatorics and Linear Algebra.Most importantly the above conversation hs given answers to two interesting questions in the research field of Analytic Number Theory.They are:Is the sum of integral powers of natural numbers always a polynomial?and Is such polynomial unique?.The most significant thing in this article is the recurrence relation given by fifth equation which will enable us to write the formulas for the sum of any positive integral powers of first $\mathrm{n}$ - natural numbers in terms of $n$.In the context of future research one can derive some more generalized results by using the Bernoulli's polynomial and some fundamental principles in Real and Complex Analyses.

\section{REFERENCES}

1. DohyoungRyang, Tony Thompson (2012), "Sum of positive integral powers "August 2012 Mathematics Teacher 106 (1):71:77.

2. Janet Beery (2010), "Sum of powers of positive integers -conclusion", Convergence,July 2010,MAA (Mathematics Teachers America)

3. Do Tan Si,"The PowersSums, Bernoullinumbers, Bernoulli Polynomials Rethinked", Applied mathematics 10.03(2019):100-112, Scientific Research 
4. Reznick,Bruce and Rouse j,"On the sums of two cubes", International Journal of number theory,7(2011),1863-1882,MR 2854220

5. Mathematical Masterpieces, by Arthur Knoebel, et al., Springer (2007), ISBN 978-0-387-33060-0. An English translation of the Potestatum appears on pp 32-37 (from the French in the 1923 Grands Écrivains edition). Good discussion is had just before and after the Potestatum itself.

6. "Pascal's Formula for the Sums of Powers of the Integers", by Carl B. Boyer, Scripta Mathematica 9 (1943), pp 237-244. Good historical background on antecedents to Pascal work on sums of powers. Contains an English translation of some of the Potestatum.

7. Pascal's Arithmetical Triangle, by A. W. F. Edwards, The Johns Hopkins University Press (2002, originally published 1987), ISBN 0-8018-6946-3. 